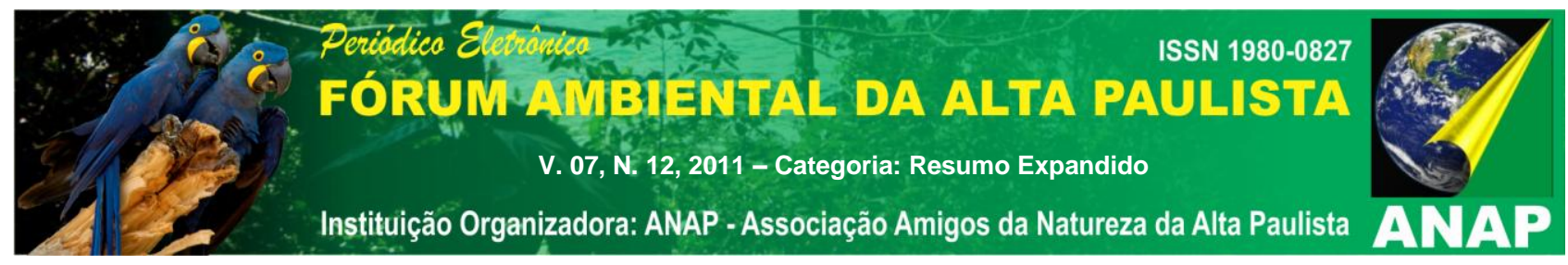

\title{
EXPANSÃO DA LEISHMANIOSE VISCERAL AMERICANA NO EXTREMO OESTE DO ESTADO DE SÃO PAULO
}

Nome do Autor (a) Principal

Lourdes Aparecida Zampieri D'Andrea

Nome (s) do Co-autor (a) (s)

Renata Corrêa Yamashita

Mariza Menezes Romão

Nome (s) do Orientador (a) (s)

Lourdes Aparecida Zampieri D'Andrea

Instituição ou Empresa

Instituto Adolfo Lutz - Centro de Laboratório Regional - Presidente Prudente V

Instituição (s) de Fomento

Programa de Aprimoramento Profissional - Fundação do Desenvolvimento Administrativo (PAP/FUNDAP)

E-mail de contato

zampieri@ial.sp.gov.br

Palavras-chave

Leishmaniose Visceral Americana, Expansão, Extremo Oeste do Estado de São Paulo 


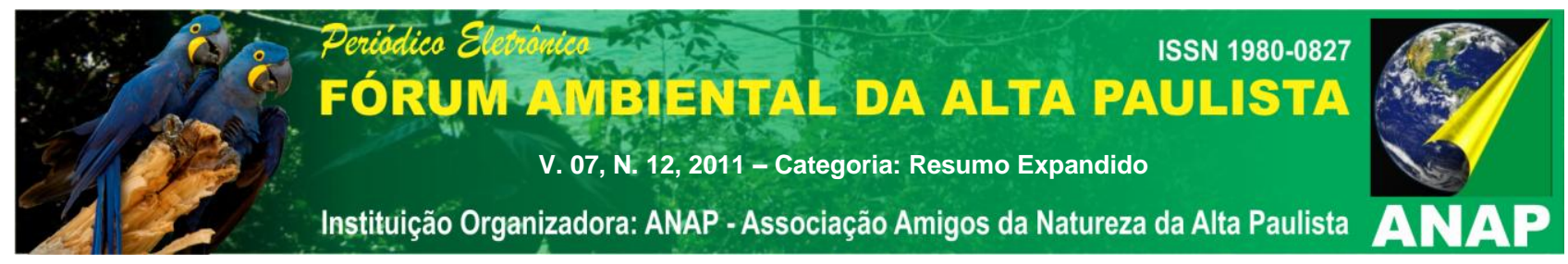

\section{INTRODUÇÃO}

As leishmanioses são consideradas importantes zoonoses que podem acometer 0 homem, quando este entra em contato com o ciclo de transmissão do parasito, transformando-se, portanto em uma antropozoonose (WHO, 2005). São um complexo de doenças causadas por protozoários do gênero Leishmania. A Leishmaniose Visceral Americana (LVA) ou calazar é causada por uma única espécie do subgênero Leishmania: a Leishmania (Leishmania) chagasi (MARZOCHI \& MARZOCHI, 1994).

Diferentes espécies de flebotomíneos transmitem a LVA, sendo a Lutzomyia longipalpis, a principal espécie envolvida nas Américas, presente em praticamente todas as áreas de ocorrência da Leishmaniose (IBGE). O ciclo biológico do L. longipalpis processa-se no ambiente terrestre, em locais úmidos, sombreados e ricos em matéria orgânica. Somente as fêmeas são hematófagas obrigatórias, sugando uma gama de animais vertebrados de sangue quente (MORRISON et.al, 1993). No Estado de São Paulo há preferência alimentar do L. longipalpis por cães (CAMARGO-NEVES, 2007). Apesar da espécie ocorrer durante todo o ano, existe variação sazonal da densidade de L. longipalpis, dependendo da região geográfica e de alguns fatores como temperatura, umidade, chuva e velocidade dos ventos (FELICIANGELI, 1987).

O padrão de transmissão da doença sofreu mudanças importantes. Inicialmente, predominava em ambientes rurais e áreas periurbanas e, posteriormente, em centros urbanos como Rio de Janeiro (RJ), Corumbá (MS), Belo Horizonte (MG), Araçatuba (SP), Palmas (TO), Três Lagoas (MS) e Campo Grande (MS). A expansão e a adaptação do vetor aos ecótopos urbanos vêm ocorrendo de forma lentamente, inicialmente sendo registrado em municípios contíguos à Araçatuba e depois naqueles que estabeleceram fluxo migratório de pessoas e mercadorias com os municípios da região de Araçatuba. Posteriormente, foi detectada em outras regiões administrativas como a de Bauru (SP), Marília (SP) e Presidente Prudente (SP), seguido pelos grandes eixos rodoviários e ferroviários.

A luta antivetorial é realizada por meio de atividades de vigilância epidemiológica como: levantamento entomológico, pesquisa entomológica de foco e em unidades fixas, notificação de insetos incômodos pela população; atividades de prevenção e controle, 


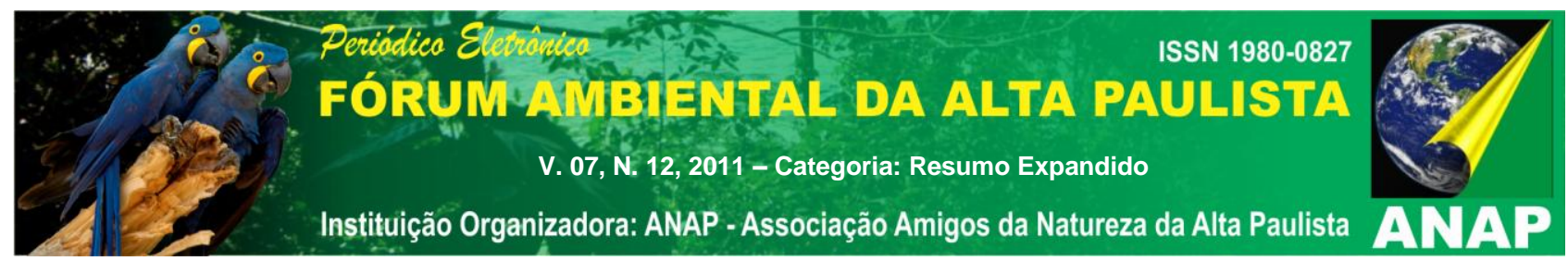

como manejo ambiental, controle químico e atividades educativas. Outro ponto fundamental do Programa de Vigilância e Controle da Leishmaniose Visceral Americana (PVCLVA) é o controle do reservatório canino por meio de inquéritos sorológicos amostral e censitário em municípios em transmissão (BRASIL/MS, 2006).

Geralmente a enzootia canina tem precedido a ocorrência de casos humanos e a infecção em cães tem sido mais prevalente do que no homem (BRENER, 1957). Por isso é de grande importância conhecer a prevalência canina infectada de uma dada região, para que o conjunto de medidas adotadas seja efetivo para redução da incidência da doença, auxiliando assim, as ações propostas pelo PVCLVA. Em virtude das características epidemiológicas, expansão e endemicidade da doença na região, torna-se necessária o conhecimento da dinâmica da disseminação da doença a fim de viabilizar o controle desta endemia através de medidas sócioeducativas e de políticas públicas.

\section{OBJETIVO GERAL}

Análise da expansão da Leishmaniose Visceral Americana no extremo Oeste do Estado de São Paulo.

\section{OBJETIVOS ESPECÍFICOS}

O estudo teve como objetivo efetuar uma análise espaço-temporal da prevalência canina de anticorpos circulantes para Leishmania sp e sua correlação com a incidência de casos humanos, bem como a importância da adoção de medidas sócioeducativas e educação ambiental como ferramentas para a promoção de mudanças do padrão de comportamento cultural visando a eliminação de criadouros do vetor da LVA e consequêntemente o controle da doença.

\section{METODOLOGIA}

A metodologia empregada no presente estudo foi um levantamento retrospectivo de dados retirados de livro de registro e Sistema de Informação e Gestão Hospitalar (SIGH) pertencentes ao Centro de Laboratório Regional - Instituto Adolfo Lutz de Presidente Prudente V (CLR-IAL-PP V) e revisão de literatura sobre o tema. 


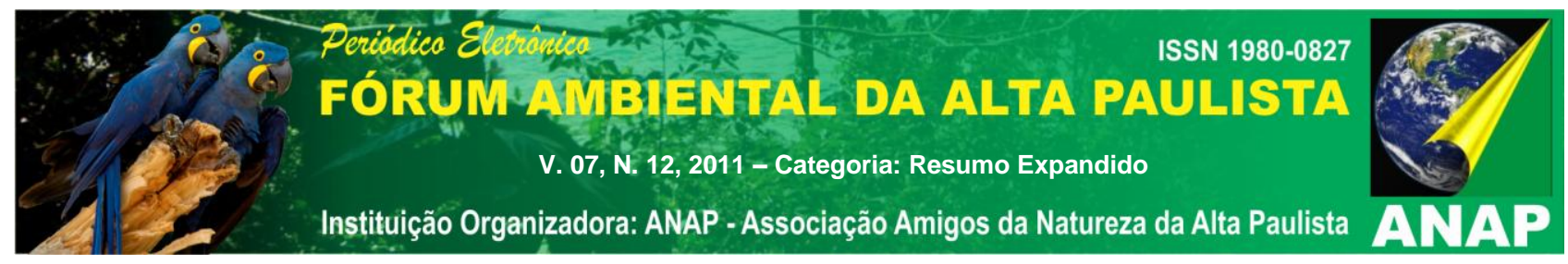

\section{RESULTADO}

A região de Presidente Prudente localizada no extremo Oeste do Estado de São Paulo (ESP) possui 45 municípios e destes, 13 tem transmissão canina e humana de Leishmaniose Visceral Americana (LVA), representando 24,1\% do total de 54 municípios paulistas notificados com casos autóctones da doença. A região conta com 194 casos notificados de 2007 a 2010, representando 22,29\% do total de casos no ESP no período (GOMES et al, 2010; CVE, 2011). No referido período, o CLR-IAL-PP V analisou um total de 53.546 cães pertencentes a municípios da micro-região de Dracena, Presidente Venceslau e Presidente Prudente, sendo que destes municípios, os que apresentaram os maiores percentuais de positividade foram Dracena $(28,77 \%)$, Panorama $(28,04 \%)$, Paulicéia (28,04\%) e Santa Mercedes (27,5\%).

Em 2003, os municípios de Dracena e Nova Guataporanga foram os primeiros a detectarem a presença de L. longipalpis, seguido em 2004 por Tupi Paulista, Junqueirópolis, Monte Castelo, Flora Rica, Santa Mercedes e São João do Pau D’Alho. Em 2005, todos os municípios pertencentes a micro-região de Dracena já tinham registrado a presença de flebotomíneos, sendo que a transmissão canina e humana nos doze municípios da micro-região de Dracena foi detectada de um a três anos após a detecção do vetor, inclusive se expandindo para municípios limítrofes, como é o caso do município de Presidente Venceslau que houve detecção do vetor em janeiro de 2008 (D’ANDREA et. al, 2009); 2009 em Caiuá, Presidente Epitácio, Presidente Prudente e Santo Expedito; 2010 no município de Teodoro Sampaio e 2011 nos municípios de Alfredo Marcondes, Álvares Machado e Martinópolis. A expansão do vetor da LVA tem ocorrido na região e consequêntemente a disseminação da doença também.

Em relação ao manejo ambiental, torna-se necessário a implantação de educação ambiental, tanto nos municípios onde a doença ainda não foi diagnosticada quanto nos que já tem o problema instalado. A questão do manejo ambiental somada a precárias condições socioeconômicas, tem se mostrado como ponto frágil e de difícil solução para o programa, pois a conscientização envolve mudanças de procedimentos há muito tempo empregado pela população, como por exemplo, a utilização de matéria orgânica como 


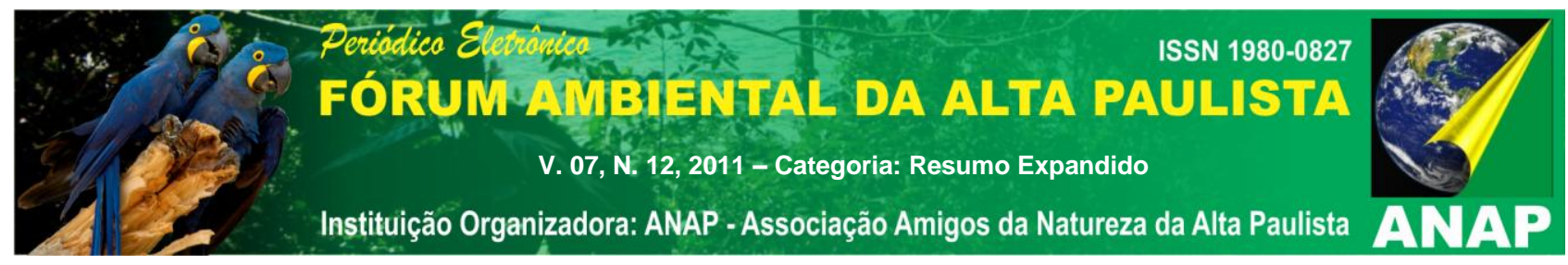

adubo natural, sendo este, substrato para o ciclo biológico do vetor, favorecendo desta forma a proliferação de flebotomíneos. A mudança do padrão de comportamento cultural esperada em função da leishmaniose só ocorrerá com o tempo, por meio desta educação, cujo objetivo é a formação de indivíduos ecoalfabetizados em todos os seguimentos, desde a infância até os profissionais atuantes. A importância do manejo adequado do solo para o controle do vetor da LVA e controle da doença, também foram descritos por Lindoso e Goto (LINDOSO et al, 2007). Barão et al. (2005) em estudo de prevalência da LVA em Araçatuba (SP), afirmaram que o favorecimento da doença pelas precárias condições socioeconômicas e ambientais, intensifica a incidência da doença. Carvalho (2005) relatou condições semelhantes pelas quais favoreceram a proliferação de flebotomíneos, possibilitando a transmissão da LVA.

\section{CONSIDERAÇÕES FINAIS}

O controle da LVA poderá ser efetuado de maneira eficaz através da implantação da educação ambiental em todos os municípios, visando a eliminação de criadouros do vetor, bem como da posse responsável do cão que é o principal reservatório da doença.

\section{REFERÊNCIAS}

BARÃO SC., CAMARGO-NEVES VLF., RESENDE MR., SILVA LJ. Human Asymptomatic Infection in Visceral Leishmaniasis: A Seroprevalence Study in an Urban Area of Low Endemicity. Preliminary Results. American Journal of Tropical Medicine and Hygiene, 2007, v. 77, 1051-1053.

BRASIL. Ministério da Saúde. Secretaria de Estado da Saúde. Manual de Vigilância e Controle da Leishmaniose Visceral Americana no Estado de São Paulo. São Paulo-SP, 2006.

BRENER Z. Calazar canino em Minas Gerais, 1957. Belo Horizonte, Universidade Federal de Minas Gerais, Faculdade de Medicina [Master's thesis].

CAMARGO-NEVES VLF. de. A Leishmaniose Visceral Americana no estado de São Paulo: situação atual. BEPA dez 2007, 4, 48. 


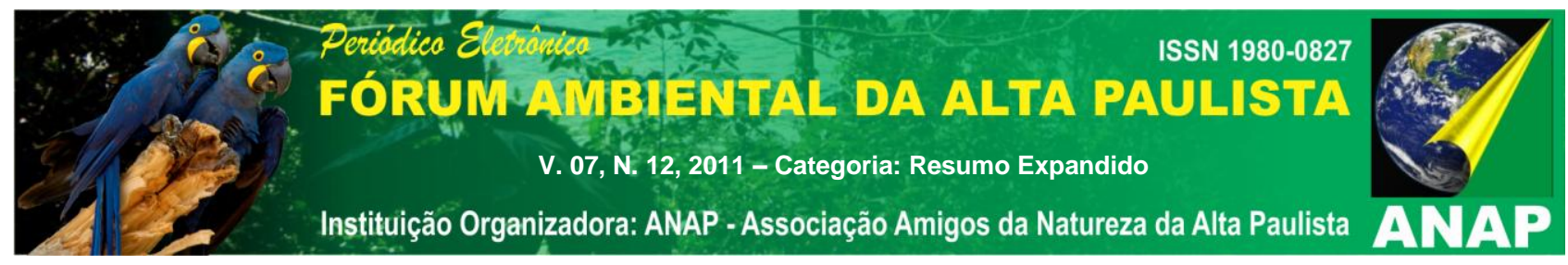

CARVALHO MR. Eco-epidemiologia da leishmaniose visceral americana na zona da mata norte de Pernambuco. Rio de Janeiro, Fundação Osvaldo Cruz, Centro de Pesquisa Aggeu Magalhães, Departamento de Saúde Coletiva, 2005. [Master's thesis].

CENTRO DE VIGILÂNCIA EPIDEMIOLÓGICA. (www.cve.saude.sp.gov.br) acesso: $15 / 09 / 2011$

D'ANDREA LAZ, CAMARGO-NEVES VLF, SAMPAIO SMP, KRONKA SN, SARTOR IF. American visceral leishmaniasis: disease control strategies in Dracena microregion in Alta Paulista, SP, Brazil. J Venom Anim Toxins incl Trop Dis. 2009;15(2):306.

FELICIANGELI MD. Ecology of sadflies (Diptera: Psychodidae) in a restricted focus of cutaneous leismaniasis in Northen Venezuela. III - Seasonal fluctuation. Mem. Inst. Osvaldo Cruz, Rio de Janeiro, 1987, 82, 167-176.

Gomes, L.H.; TOLEZANO, J. E.; HIRAMOTO, R. M.; CIARAVOLO, R. M.; RANGEL, O.; TAKAOKA, N.; REICHMANN, M. L. A. B.; SICILIANO, M. M.; CRUZ, L. L.. Classificação epidemiológica dos municípios segundo o Programa de Vigilância e Controle da Leishmaniose Visceral Americana no Estado de São Paulo, atualizado em maio de 2010. BEPA. Boletim Epidemiológico Paulista (Online) ${ }^{\mathrm{JCR}}$, v. 7, p. 21-40, 2010.

IBGE. Mapas Temáticos (http://www.ibge.gov.br/mapas_ibge/tem.php).

LINDOSO JAL., LIMA VP., GOTO H., VERGARA MPP., CELESTE BJ. Correlação clínica da resposta sorológica para Leishmania sp em pacientes com Aids atendidos no Instituto de Infectologia Emílio Ribas. In: REUNIÃO DE PESQUISA APLICADA EM DOENÇA DE CHAGAS E LEISHMANIOSE, 23,Uberaba, 2007. Resumos... Uberaba, 2007.

MARZOCHI MCA., MARZOCHI KBF. Tegumentary and Visceral Leishmaniasis in BrazilEmerging Anthroponosis and Possibilies of their Control. Cad. Saúde Pública, 1994, 10, 2, 359-378.

MORRISON AC., FERRO C., TESH RB. Host preference of the fly Lutzomyia longipalpis at an endemic focus of American Visceral Leishmaniasis in Colombia. Am. J. Trop. Med. Hyg. 1993, 49, 68-75.

WORLD HEALTH ORGANIZATION. Division of control of tropical disease. Leishmaniasis control. Geographical distribution. $2005 . \quad$ WHO/CTD. (http://www.who.int/ctd/html/leisgeo.html). 